\title{
Mechanical-Thermal Noise in Micromachined Acoustic and Vibration Sensors
}

\author{
Thomas B. Gabrielson
}

\begin{abstract}
Since the introduction of the micromachining process, wherein mechanical structures are etched from blocks of silicon, a number of very small acoustic and vibration sensors have been built. This size reduction is attractive for many applications but the small moving parts are especially susceptible to mechanical noise resulting from molecular agitation. For sensors designed for small-signal applications (microphones and hydrophones, for example), this mechanical-thermal noise is often one of the limiting noise components. While this component is often neglected in design and analysis, it is relatively easy to estimate, since, like electrical-thermal noise, the magnitude of mechanical-thermal noise depends only on temperature and the magnitude of mechanical damping. This paper reviews several techniques for calculating the mechanical-thermal noise in acoustic and vibration sensors in general and in micromachined sensors in particular.
\end{abstract}

\section{INTRODUCTION}

$\mathrm{T}$ HE micromachining process has made possible the construction of a variety of miniature small-signal accelerometers and acoustic pressure sensors (for example, [1]-[3]). Ranging from simple capacitive pressure sensors [4] to accelerometers that measure the proof-mass displacement by electron tunneling [5], these sensors are attractive for many space-limited applications. However, in gases at normal pressures or in liquids, the small moving parts are especially susceptible to mechanical noise resulting from molecular agitation. If the sensor is intended for low-level signals, this mechanical-thermal noise can be the limiting noise component.

Mechanical-thermal noise is not a new concept but its effects are frequently "rediscovered" when a sensor technology is pushed to the limit of its sensitivity. Documented examples range from the mirrored galvanometer [6] to condensor microphones [7] and gravity-wave detectors [8]. One of the more well-known mechanisms for mechanical-thermal noise is Brownian Motion. Here, the agitation of an observable object is caused by molecular collisions from a surrounding gas or liquid and the agitation is directly related to the fluid's viscosity [9]. In fact, any molecular agitation even through solid structures like springs and supports can cause random motion of an object. Often, it is assumed that the preamplifier noise dom-

Manuscript received December 2, 1991; revised July 24, 1992. This work was supported by the Office of Naval Research. The review of this paper was arranged by Associate Editor S. D. Senturia.

The author is with the Naval Air Warfare Center, Code 5044, Warminster, PA 18974.

IEEE Log Number 9208061. inates the sensor noise floor [10]; while this is true in some cases, this assumption is particularly dangerous with microminiature sensors for which mechanical-thermal noise can sometimes set a much higher noise floor.

\section{Mechanical-Thermal Noise}

The thermodynamically proper equation of motion for the displacement $z$ of a damped harmonic oscillator with mass $m$, spring constant $k$, and mechanical resistance $R$, is

$$
m \frac{d^{2} z}{d t^{2}}+R \frac{d z}{d t}+k z=f_{n}(R, t)
$$

The presence of damping in the system suggests that any oscillation would continue to decrease in amplitude forever. Even the small, random jitter caused by molecular motion would decay. Inclusion of the fluctuating force $f_{n}$ prevents the system temperature from dropping below that of the system's surroundings.

The presence of the damping term in the equation requires that the fluctuating force be present as well. The damper provides a path for energy to leave the mass-spring system but this path works both ways: Random thermal agitation from the environment also affects the oscillator's motion. This is the essence of the Fluctuation-Dissipation Theorem [11]. If there is a mechanism for dissipation in a system, then there will also be a component of fluctuation in that system directly related to that dissipation.

In practice, there are two tools for analyzing equilibrium fluctuations: the Equipartition Theorem and the Nyquist Relation. According to Equipartition [12], [13], if any collection of energy storage modes is in thermal equilibrium, then each mode will have an average energy equal to $\frac{1}{2} k_{B} T$ where $k_{B}$ is Boltzmann's constant $(1.38 \times$ $10^{-23} \mathrm{~J} / \mathrm{K}$ ) and $T$ is the absolute temperature. A mode of energy storage is one in which the energy is proportional to the square of some coordinate; e.g., kinetic $\left(\frac{1}{2} m v^{2}\right)$, spring potential $\left(\frac{1}{2} k x^{2}\right)$, electrical potential $\left(\frac{1}{2} C V^{2}\right)$, rotational-kinetic $\left(\frac{1}{2} I \omega^{2}\right)$. Consequently, the mean-square displacement of a mass-spring oscillator resulting from thermal agitation is

$$
\frac{1}{2} k\left\langle x^{2}\right\rangle=\frac{1}{2} k_{B} T \quad[\mathrm{~J}]
$$

where $\left\langle x^{2}\right\rangle$ can be taken as the average of the spectral density of $x^{2}$ over all frequencies. 
Nyquist's Relation [13], [14] gives the spectral density of the fluctuating force related to any mechanical resistance

$$
F=\sqrt{4 k_{B} T R} \quad[\mathrm{~N} / \sqrt{\mathrm{Hz}}]
$$

or the fluctuation pressure related to any acoustic resistance $\left(R_{a c s}=R / S^{2}\right.$ where $S$ is the area of the active face)

$$
p=\sqrt{4 k_{B} T R_{a c s}} \quad[\mathrm{~Pa} / \sqrt{\mathrm{Hz}}] .
$$

This is a direct physical analog of Johnson noise related to electrical resistance. (This is not surprising since Johnson noise is produced by scattering of free electrons by mechanical-thermal vibration of the conductor's solid lattice [15].) Nyquist's Relation holds even if the resistance is a function of frequency [11]. This result is derived directly from equipartition: A system mode having $\frac{1}{2} k_{B} T$ broadband energy is equivalent to the damper in the system having an associated force generator with $\sqrt{4 k_{B} T R}$ spectral density.

Any mechanical system in thermal equilibrium, no matter how complex, can be analyzed for mechanical-thermal noise by adding a force generator alongside each damper. Fig. 1 shows the inclusion of such a noise force for a simple mass-spring oscillator and Fig. 2 illustrates the noise forces for a compound mass-spring system. Notice especially in Fig. 2 that the noise force acts at both ends of the damper. (The action on the frame in Figs. 1 and 2 is inconsequential for these analyses and so is omitted.) Since the noise mechanisms are usually uncorrelated, the system would be analyzed for each noise force separately. Then the total noise would be computed by adding the squares of the individual noise components and taking the square root of the sum.

Often, the most convenient approach to noise analysis in complicated systems is to draw the electrical equivalent circuit and use a software circuit-analysis program to determine the noise response. In fact, it is hard to ignore mechanical-thermal noise this way because the mechanical dampers translate to electrical resistors and the Johnson noise of resistors is well known.

\section{A. The Simple Accelerometer}

The generic accelerometer sensor is shown schematically in Fig. 1. When the case is exposed to some acceleration, the interior mass moves relative to the case. This relative displacement is translated to an electrical output signal. Also shown in Fig. 1 is the equivalent noise force $F_{n}$. Of course, the noise force also acts on the sensor case through the other end of the damper but that is not important here. In the frequency domain, the signal excitation displacement is $Y(f)$ and the response is $Z(f)$. To get the noise response, set the signal $Y$ to zero and solve for $Z_{n}$ in terms of $F_{n}$; to get the signal response, set $F_{n}$ to zero and solve for $Z_{s}$ in terms of the case displacement $Y$.

Solving the simple accelerometer for the noise response gives

$$
\left|Z_{n}(f)\right|=\sqrt{4 k_{B} T R} G(f) / k \quad[\mathrm{~m} / \sqrt{\mathrm{Hz}}]
$$
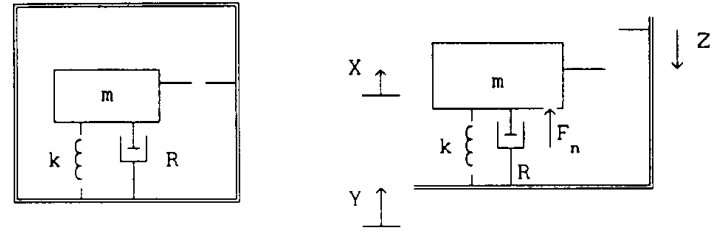

Fig. 1. Schematic diagram of a simple accelerometer including displacement coordinates and free-body diagram.

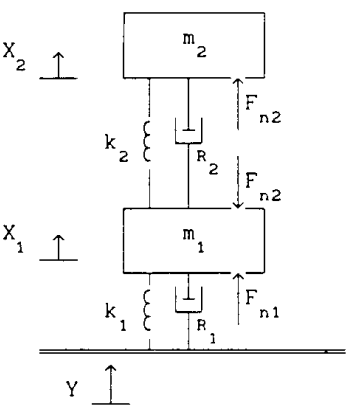

Fig. 2. Illustration of addition of noise forces to a two-mass-spring system.

where

$$
G(f)=1 / \sqrt{\left[1-\left(f / f_{0}\right)^{2}\right]^{2}+\left(f / f_{0}\right)^{2} / Q^{2}}
$$

and $4 \pi^{2} f_{0}^{2}=\omega_{0}^{2}=k / m$. In the accelerometer limit $(f$ $\ll f_{0}$ ), the noise displacement becomes

$$
\left|Z_{n}(f)\right|=\sqrt{4 k_{B} T R} / k
$$

which can also be expressed in terms of the $Q$ of the oscillator (since $Q=\omega_{0} m / R$ )

$$
\left|Z_{n}(f)\right|=\sqrt{4 k_{B} T / \omega_{0} k Q}=\sqrt{4 k_{B} T / \omega_{0}^{3} m Q} .
$$

The signal response is

$$
\left|Z_{s}(f)\right|=\left(f / f_{0}\right)^{2} G(f)\left|Y_{s}\right|
$$

but $\omega^{2}\left|Y_{s}\right|$ is the magnitude of the input acceleration, $a_{s}$ (in meters per square second per root hertz), so

$$
\left|Z_{s}(f)\right|=a_{s} G(f) / \omega_{0}^{2} .
$$

$G(f)=1$ in the accelerometer limit.

The signal-to-noise ratio (SNR) at any frequency is then

$$
\left|Z_{s} / Z_{n}\right|^{2}=a_{s}^{2} m Q / 4 k_{B} T \omega_{0}
$$

Here, the "signal"' is taken to be the power spectral density of the ambient noise of the environment. This is appropriate for a sensor designed for optimum reception of real signals in this ambient; if, however, the real signals are guaranteed never to approach the environmental ambient, then the minimum real signal level should be used. (That could be a single-frequency level rather than a spectral density in which case the noise power in the analysis bandwidth should be used instead of noise spectral density.) 

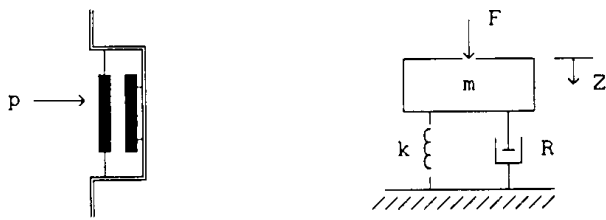

Fig. 3. Schematic diagram of simple pressure sensor including displacement coordinate and applied force.

From (11), it is apparent that the SNR can be improved by increasing the $Q$, reducing the resonance frequency $\omega_{0}$, or increasing the mass $m$ (which also lowers $\omega_{0}$ ). Increasing the mass runs counter to miniaturization. Lowering $\omega_{0}$ far enough so that it is within the band of expected signals is usually not prudent because this introduces a nonlinear phase into the system response. Increasing the $Q$ can cause problems also: if the oscillator has a high $Q$, then out-ofband oscillations can be large (as much as $Q$ times the inband amplitude) and the mechanical system must have enough dynamic range to handle these large movements. Also, a high- $Q$ system introduces long, oscillatory tails onto any rapid level changes.

\section{B. The Simple Pressure Sensor}

In the simple pressure sensor, the moving mass is directly exposed to the incident acoustic pressure and the displacement of this mass is measured. Such a sensor is shown schematically in Fig. 3. For an arbitrary force $F$, the displacement response $Z$ is

$$
|Z|=F G(f) / k \text {. }
$$

For signal, the force $F=p_{s} S$, where $S$ is the area of the transducer face and $p_{s}$ is the spectral density of the signal pressure; for noise, the force $F=\sqrt{4 k_{B} T R}$, so the signalto-noise ratio is

$$
\left|Z_{s} / Z_{n}\right|^{2}=\left(p_{s} S\right)^{2} / 4 k_{B} T R=Q\left(p_{s} S\right)^{2} / 4 k_{B} T \omega_{0} m .
$$

In this case, the signal-to-noise ratio can be improved by increasing the area, increasing the $Q$, reducing the resonance frequency, or reducing the mass. Reduction of the mass would seem to favor microminiaturization but the $\omega_{0} m$ product goes as $\sqrt{m}$, which makes this dependence weak, whereas the dependence on area is as area squared.

The foregoing analyses apply to sensors that are wellrepresented by a single mass-spring system. While this is adequate for many hydrophones, microphones, and vibration sensors, some designs are more complex. The solution procedure for more complicated systems is more tedious but it is, in principle, identical to the simpler systems: Each damper in the system is given its own noise-force generator.

\section{Dissipation Mechanisms}

One of the keys to evaluating mechanical-thermal noise is understanding the sources of dissipation in the system. In terms of fluctuations, any mechanism that allows en- ergy to escape from the orderly motion of the sensor counts as dissipation. These mechanisms include mechanical damping in the spring and supports, viscous drag, acoustic reradiation, electrical leakage, and magnetic eddy-current damping. (In some cases, the deterministic effects of damping can be reduced by electromechanical feedback but this in no way improves the ratio of signal to thermal noise. It is the real damping that determines the thermal fluctuations, not the apparent damping after feedback.)

Many times, measurements of a vibrating system's dominant mass and $Q$ are simple; the resistance and, therefore, the fluctuating force can be calculated from these measurements. In other cases, the damping mechanism is simple enough to permit direct calculation.

Once the relevant resistance has been calculated or measured, the signal-to-noise ratio can be computed from (11) or (13). Alternatively, the equivalent noise pressure (or acceleration) can be computed for comparison with the lowest signal level anticipated. The equivalent noise pressure (in $\mathrm{Pa} / \sqrt{\mathrm{Hz}}$ ), is equal to the spectral density of the fluctuating force (3) divided by the area of the sensor diaphragm (or, directly from (4)).

For capacitive sensors, the very thin gaps that permit low polarization voltage and high capacitance per unit area also lead to squeeze-film damping-the viscous loss associated with squeezing the fluid out from between moving surfaces [16]. Squeeze-film damping can easily dominate the dissipation mechanisms for gaps of several micrometers. For two parallel disks of area $S$, with average spacing $h_{0}$, the equivalent mechanical resistance is

$$
R_{\text {film }}=3 \mu S^{2} / 2 \pi h_{0}^{3} \quad[\mathrm{~N} \cdot \mathrm{s} / \mathrm{m}]
$$

where $\mu$ is the fluid's viscosity $\left(18 \times 10^{-6} \mathrm{~kg} / \mathrm{m} \cdot \mathrm{s}\right.$ for air at $20^{\circ} \mathrm{C} ; 10^{-3} \mathrm{~kg} / \mathrm{m} \cdot \mathrm{s}$ for water at $20^{\circ} \mathrm{C}$ ). Notice the strong dependence on the spacing.

If one disk is perforated, the damping can be reduced considerably as the fluid can escape through the perforations instead of being squeezed out the disk edges. In this case, the mechanical resistance is given approximately by [17]

$$
R_{\mathrm{perf}}=\frac{12 \mu}{N \pi h^{3}} G(A) S^{2}
$$

where $N$ is the total number of holes in the perforated plate, $A$ is the fraction of open area in the plate, and

$$
G(A)=\left[\frac{A}{2}-\frac{A^{2}}{8}-\frac{\ln A}{4}-\frac{3}{8}\right] .
$$

This expression assumes that the fluid flows only through the perforation holes and not out beyond the edges of the plates; consequently, the limit as $A$ goes to zero is not the simple squeeze-film expression, (14). A more complex but more accurate theoretical expression is given by Zuckerwar [18]; however, for many practical applications a good approximation consists of the parallel combination of the resistances given by (14) and (15). 
Radiation resistance can be a significant dissipation mechanism above $10 \mathrm{kHz}$. The mechanical radiation resistance for a simple pressure sensor is approximated closely (for $k a<1$ ) by [19]

$$
R_{r}=\rho c \pi a^{2}(k a)^{2} / 4 \quad[\mathrm{~N} \cdot \mathrm{s} / \mathrm{m}]
$$

where $k$ is the acoustic wavenumber $(\omega / c), \rho$ and $c$ are the density and sound speed of the fluid, and $a$ is the radius of the piston. (The convention $e^{+i \omega t}$ is assumed.) Occasionally, the noise corresponding to the radiation resistance is taken to be the only relevant component of thermal-agitation noise at normal pressures [20], [21]; this is certainly not true because the radiation impedance expression given above ignores the effects of viscosity. For sensors operating below several tens of kilohertz, the noise associated with film damping (14) and (15) normally overwhelms that associated with the zero-viscosity radiation resistance.

\section{Capacitive Microphone}

Noise-floor requirements for microphones can be demanding. A professional recording studio would generally be designed to keep the background noise at an A-weighted level of $25 \mathrm{~dB}$ [22], which corresponds to a spectral density of several micropascals per root hertz. Research applications might require even lower levels. An acoustic sensor for such applications must have a noise floor somewhat below these levels. Although mechanicalthermal noise is not so serious a problem in conventionally sized microphones, it is easily observed. For example, the measurements of Tarnow [7], [23], and Ngo [24] on Brüel and Kjaer condensor microphones showed that the noise floor above several hundred hertz results from mechanical-thermal noise.

In a micromachined capacitive sensor, the gap can be made very small (several micrometers at a unit cost of tens of dollars) thereby producing small sensors with a proportionately higher capacitance than conventional sensors. As discussed below, this accentuates mechanicalthermal noise especially since the damping increases rapidly with decreasing gap. In addition, heterodyne (ac bias) schemes are available (e.g., [25], [26]) which, in effect, reduce the impedance of the capacitor element to preamplifier noise currents. This can radically reduce the effect of the preamplifier current noise but does nothing about the mechanical-thermal noise. If the designer is unaware of the mechanical-thermal noise floor, unrealistic expectations can result.

Since good noise spectral measurements are rarely published for micromachined sensors, it is worth considering some of the experiments with conventional capacitor microphones. Tarnow [7], [23] and, later, Ngo [24] both performed careful spectral density measurements of noise from Brüel and Kjaer capacitor microphone cartridges. With measurements of the microphone damping and predictions by Zuckerwar [18], excellent agreement was ob- tained between predicted levels of mechanical-thermal noise and the observed noise above several hundred hertz. (These measurements were all done with dc bias and so the low-frequency noise was dominated by the preamplifier current noise interacting with the high-impedance capacitive element.)

For example, the Brüel and Kjaer 4134 ( $1 / 2$ in) capacitor microphone has been modeled and measured by several researchers [7], [18], [23], [24]. Zuckerwar [18] measured the following characteristics of the diaphragm and backplate: a backplate of radius $=3.61 \mathrm{~mm}$ with 6 holes, each having a radius of $0.508 \mathrm{~mm}$, and a backplateto-diaphragm spacing of $21 \mu \mathrm{m}$. Using a parallel combination of the resistances given by (14) and (15) (and dividing by the sensor area squared), this gives a total acoustic resistance of $207 \times 10^{6} \mathrm{~N} \cdot \mathrm{s} / \mathrm{m}^{5}$. Zuckerwar's more accurate calculation gives a value of $189 \times 10^{6} \mathrm{~N}$ $\cdot \mathrm{s} / \mathrm{m}^{5}$, while Tarnow [7] quotes a value of $154 \times 10^{6} \mathrm{~N}$ $\cdot \mathrm{s} / \mathrm{m}^{5}$ and $\mathrm{Ngo}[24]$ uses $125 \times 10^{6} \mathrm{~N} \cdot \mathrm{s} / \mathrm{m}^{5}$. Overall, the equivalent pressure noise related to these resistance values (4) would be between 1.4 and $1.8 \mu \mathrm{Pa} / \sqrt{\mathrm{Hz}}$. Tarnow measured a level of between 1.6 and $2 \mu \mathrm{Pa} / \sqrt{\mathrm{Hz}}$ from 1 to $20 \mathrm{kHz}$; Ngo measured a level of $2 \mu \mathrm{Pa} / \sqrt{\mathrm{Hz}}$ between 2 and $20 \mathrm{kHz}$. The correspondence between either measurement and the predicted range is good.

Tarnow [7] also measured the noise for the larger B\&K 4144 ( 1 in) microphone. He quotes an acoustic resistance of $18 \times 10^{6} \mathrm{~N} \cdot \mathrm{s} / \mathrm{m}^{5}$, which corresponds to an equivalent noise pressure of $0.54 \mu \mathrm{Pa} / \sqrt{\mathrm{Hz}}$, and he measured levels of from 0.4 to $0.5 \mu \mathrm{Pa} / \sqrt{\mathrm{Hz}}$. These measurements covered $100 \mathrm{~Hz}$ to $20 \mathrm{kHz}$, well beyond the natural resonance of the microphone $(5 \mathrm{kHz})$ and, when corrected for the frequency response, agreed well with the predicted value for mechanical-thermal noise alone. He also measured the temperature dependence of the noise down to liquid-nitrogen temperature $(77 \mathrm{~K})$ and found the proper linear dependence on absolute temperature for the noise power.

As an example of mechanical-thermal noise analysis for a micromachined sensor, consider the capacitive microphone described by Hohm and Hess [4]. This device has a 0.8 by $0.8 \mathrm{~mm}$ diaphragm and a 0.5 by $0.6 \mathrm{~mm}$ backplate forming an active capacitor with a $2-\mu \mathrm{m}$ gap. With air as the dielectric, the capacitance is $1.3 \mathrm{pF}$ and the squeeze-film damping resistance from (14) is about $0.1 \mathrm{~N}$ - $\mathrm{s} / \mathrm{m}$ (which corresponds to an acoustic resistance of 2.4 $\times 10^{11} \mathrm{~N} \cdot \mathrm{s} / \mathrm{m}^{5}$ ). The damping terms that results from reradiation and viscous flow around the backplate into the back volume are several orders of magnitude below the squeeze-film damping and any mechanical damping in the diaphragm will be ignored.

The rms fluctuating force on the diaphragm (3) associated with the squeeze-film damping dominates the mechanical-thermal noise and is equal to $4 \times 10^{-11}$ $\mathrm{N} / \sqrt{\mathrm{Hz}}$, which, when applied to the $0.64 \times 10^{-6} \mathrm{~m}^{2}$ diaphragm, results in an equivalent noise pressure of 60 $\mu \mathrm{Pa} / \sqrt{\mathrm{Hz}}$. This equivalent noise pressure spectral density 
corresponds to an A-weighted sound level of about $50 \mathrm{~dB}$. (The equivalent noise bandwidth of the A-weighting function is about $15 \mathrm{kHz}$.) In comparison, the ambient noise level ranges from 25 to $30 \mathrm{~dB}$ in a quiet recording studio [22] and from 30 to $50 \mathrm{~dB}$ in a quiet residential area [19]. Consequently, this microphone is not suited for recording low-level signals in quiet environments (as the authors remark).

If this microphone had been intended for, say, recording studio use, it would have to be redesigned. Since its intrinsic noise floor mimics such a high acoustic level, it is immaterial what the pressure-to-voltage transfer constant of the microphone is: the stated design is inadequate. The transfer constant (receiving sensitivity) does determine whether or not the preamplifier will dominate the noise floor. The measured transfer constant for this device is about $1 \mathrm{mV} / \mathrm{Pa}$, so the voltage noise corresponding to the mechanical-thermal noise would be 60 $\mathrm{nV} / \sqrt{\mathrm{Hz}}$. Using an inexpensive, low-noise JFET like the $2 \mathrm{~N} 4338(8-\mathrm{nV} / \sqrt{\mathrm{Hz}}$ input voltage noise, $0.8-\mathrm{fA} / \sqrt{\mathrm{Hz}}$ input current noise) as the preamplifier input stage, the mechanical-thermal noise would be the dominant component above $500 \mathrm{~Hz}$. (The total capacitance of the microphone is $6 \mathrm{pF}$.)

If an equivalent A-weighted noise level of $20 \mathrm{~dB}$ were required, the microphone would have to be modified. This level corresponds, roughly, to a uniform pressure spectral density of $2 \mu \mathrm{Pa} / \sqrt{\mathrm{Hz}}$. The acoustic resistance, then, must be no larger than $2.5 \times 10^{8} \mathrm{~N} \cdot \mathrm{s} / \mathrm{m}^{5}$. If the backplate area is assumed to be half of the diaphragm area, then the squeeze-film resistance equation (14) can be solved for the new diaphragm-to-backplate spacing, which is about $20 \mu \mathrm{m}$-ten times larger than the original spacing.

If no other changes were made, the active capacitance would be one tenth of the original capacitance and the electromechanical transformation coefficient [19], $\phi$ (ratio of diaphragm velocity to electrical current) would be one hundredth of the original value, where

$$
\phi=C_{0} V_{0} / h_{0} \quad[\mathrm{~A} \cdot \mathrm{s} / \mathrm{m}] .
$$

Here, $C_{0}$ is the active capacitance, $V_{0}$ is the bias voltage, and $h_{0}$ is the gap. The smaller value of $\phi$ would lead to greatly reduced sensitivity. If the diaphragm area were increased to maintain the original capacitance, the new diaphragm would be 2.5 by $2.5 \mathrm{~mm}$, and the polarization voltage would have to be raised by a factor of ten to maintain the original $\phi$. Also, the back volume would have to be enlarged to preserve the sensitivity. Overall, the result is a much larger sensor.

A complete noise analysis would, of course, include the effects of at least the first stage of amplification. In order to properly account for the source impedance, a complete electromechanical equivalent circuit should be drawn. One of the standard representations [19] for a capacitive sensor is shown in Fig. 4. The radiation impedance is included as $R_{r}$ and $m_{r}$; the mechanical damping,

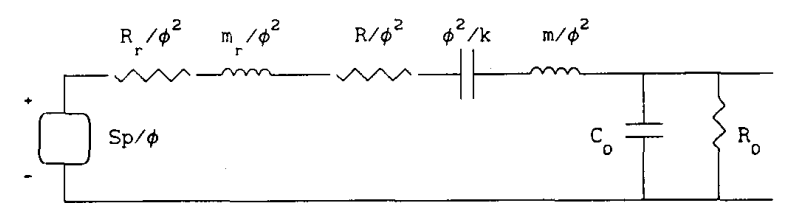

Fig. 4. Electromechanical equivalent circuit for capacitive microphone.

stiffness, and mass of the sensor are included as $R, k$, and $m$; and the electrical characteristics as $C_{0}$ and $R_{0}$. Parasitic capacitance would be added in parallel with $C_{0}$. The "voltage" driver is a function of the acoustic pressure $p$, and the transducer diaphragm area $S$.

Rather than increasing the plate spacing, a more effective way to decrease the damping is to perforate the backplate. Bergqvist et al. [17] describe such a micromachined microphone, which has a calculated noise level of $22 \mathrm{~dB}(\mathrm{~A})$ with a 2 by $2 \mathrm{~mm}$ diaphragm. While not much smaller than the $20 \mathrm{~dB}(\mathrm{~A})$ design above, a small gap ( 2 $\mu \mathrm{m})$ can be maintained, which allows a low bias voltage.

This unit has a transfer constant of $1.8 \mathrm{mV} / \mathrm{Pa}$, a working capacitance of $5 \mathrm{pF}$, and a total (including stray) capacitance of $20 \mathrm{pF}$. From estimates from electron microphotographs, the backplate appears to have about 1600 holes with a ratio of open to total area $(A)$ of about $1 / 3$. In air, then, using (15), the acoustic resistance would be $280 \times 10^{6} \mathrm{~N} \cdot \mathrm{s} / \mathrm{m}^{5}$, which results in an equivalent noise pressure of $2 \mu \mathrm{Pa} / \sqrt{\mathrm{Hz}}$ or about $22 \mathrm{~dB}(\mathrm{~A})$. This would be adequate for many low-noise applications. Because the capacitance is low (as is true for most micromachined capacitive sensors), unless a heterodyne system is used [e.g., 26], the preamplifier input current noise must be considered. With a transfer constant of $1.8 \mathrm{mV} / \mathrm{Pa}$, the mechanical-thermal noise voltage would be $4 \mathrm{nV} / \sqrt{\mathrm{Hz}}$ or just below the input voltage noise of a 2 N4338 JFET. For a total source capacitance of $20 \mathrm{pF}$, the input voltage component that results from the JFET current noise into the source impedance would be greater than $4 \mathrm{nV} / \sqrt{\mathrm{Hz}}$ at all frequencies below $1600 \mathrm{~Hz}$.

While the Bergqvist design performs well as a microphone, a similarly designed hydrophone may not be so successful. In order to keep the diaphragm from collapsing onto the backplate with increasing hydrostatic pressure, such a hydrophone would normally be fluid-filled instead of air-filled. Without a large increase in fluid-reservoir volume, the transfer constant would drop dramatically, but there is an even more fundamental change in the mechanical-thermal noise. Using the same physical parameters for the diaphragm and backplate as above, but substituting an electrical-grade silicone oil (kinematic viscosity of 50 centistokes or dynamic viscosity of $0.046 \mathrm{~N}$ $\cdot \mathrm{s} / \mathrm{m}^{3}$ ) for the air increases the acoustic resistance to 720 $\times 10^{9} \mathrm{~N} \cdot \mathrm{s} / \mathrm{m}^{5}$. This results in an equivalent pressure noise of $107 \mu \mathrm{Pa} / \sqrt{\mathrm{Hz}}$, a level high enough to interfere with ambient noise measurements in the ocean above several hundred hertz [27]. Lower viscosity oils are available but leakage currents, ion migration, and depolarization can prevent their use [28] especially with dc bias. 


\section{Electron-TunNeling ACCELERometer}

Accelerometers are often used to measure motion of solid surfaces and to measure directional components of acoustic and nonacoustic motion in fluids. In the case of an underwater acoustic sensor, a minimum detectable signal of better than $10^{-7} \mathrm{~m} / \mathrm{s}^{2} / \sqrt{\mathrm{Hz}}(10 \mathrm{ng} / \sqrt{\mathrm{Hz}})$ might be required [27] for detecting low-level signals against a quiet ocean ambient level. While capacitive accelerometers have been built, accelerometers that use electron tunneling to measure the displacement of the proof mass with respect to the case have some advantages. Low-frequency noise for tunneling goes as $1 / f$ instead of $1 / f^{2}$ as for a capacitor. In addition, the displacement sensitivity is high. The tunneling current $I_{t}$ is exponentially sensitive to the separation $x$

$$
I_{t}=I_{0} \exp \left(-x / x_{0}\right) \quad[\mathrm{A}]
$$

where $I_{0}$ is a function of the voltage $V_{b}$ applied between the conductors and

$$
x_{0}=1 / \alpha \sqrt{\Phi} \quad[\mathrm{m}]
$$

where $\alpha=1.025 \times 10^{10} \mathrm{~m}^{-1} \cdot \mathrm{eV}^{-1 / 2}$ and $\Phi$ is the effective work function of the surfaces [29]. For gold to gold, $\Phi$ is about $0.5 \mathrm{eV}$ and $x_{0}$ is about a tenth of a nanometer [5]. Therefore, the tunneling current changes by a factor of about three for a displacement change of a tenth of a nanometer. The small-signal displacement-to-current transfer constant is given by the derivative of (19), or

$$
|d I / d x|=I_{t} / x_{0} \quad[\mathrm{~A} / \mathrm{m}] .
$$

Since $x_{0}$ is so small, the tunneling current is extremely sensitive to displacement. The response to displacement, however, is strongly nonlinear so electromechanical feedback is normally used to make the output linear (and to stabilize the tunneling gap).

As an example, consider the electron-tunneling accelerometer described by Kenny et al. [5]. This is a micromachined device with a mass of $30 \times 10^{-6} \mathrm{~kg}$ and a spring constant of $60 \mathrm{~N} / \mathrm{m}$ (thus a resonance frequency of 225 $\mathrm{Hz}$ ). The gold-to-gold gap is maintained so that the average tunneling current is $1.3 \mathrm{nA}$ with an applied bias voltage of $100 \mathrm{mV}$. According to (21), the displacementto-current sensitivity is $9.4 \mathrm{~A} / \mathrm{m}$. The dynamic response can be calculated from (10) and is equal to $9.8 / \omega_{0}^{2}$ meters per $g$ of acceleration below the resonance frequency. Coupled with the displacement current sensitivity, the overall transfer constant is $4.6 \times 10^{-5} \mathrm{~A} / \mathrm{g}$. With a JFETinput preamplifier (e.g., 2N4338) having an input current noise of the order of $10^{-15} \mathrm{~A} / \sqrt{\mathrm{Hz}}$, this suggests a minimum sensitivity of $10^{-10}$ to $10^{-11} \mathrm{~g} / \sqrt{\mathrm{Hz}}$, which would be remarkable performance.

Because tunneling involves random emission of electrons across the gap, a shot-noise component [13] must also be considered

$$
i_{s}=\sqrt{2 q I_{t}} \quad[\mathrm{~A} / \sqrt{\mathrm{Hz}}]
$$

where $q$ is the charge on an electron $\left(1.6 \times 10^{-19} \mathrm{C}\right)$. For a tunnel current of $1.3 \mathrm{nA}$, the shot-noise component is 2 $\times 10^{-14} \mathrm{~A} / \sqrt{\mathrm{Hz}}$-an order of magnitude higher than the hypothetical preamplifier noise. This would reduce the expected minimum sensitivity to $4 \times 10^{-10} \mathrm{~g} / \sqrt{\mathrm{Hz}}$, still well below the $10 \mathrm{ng} / \sqrt{\mathrm{Hz}}$ that might be required for an underwater acoustic sensor.

The force-balance feedback for this accelerometer is applied by electrostatic force through parallel plates, 7 $\mathrm{mm}$ by $11 \mathrm{~mm}$, spaced $50 \mu \mathrm{m}$ apart. Since the plates are solid, the squeeze-film damping can be calculated from (14) for air between the plates. The resulting mechanical damping is $0.41 \mathrm{~N} \cdot \mathrm{s} / \mathrm{m}$ (which corresponds to a $Q$ of $0.1)$. From (7), the displacement noise below the resonance frequency is $1.3 \times 10^{-12} \mathrm{~m} / \sqrt{\mathrm{Hz}}$. The equivalent acceleration noise is $2.7 \times 10^{-7} \mathrm{~g} / \sqrt{\mathrm{Hz}}$, much greater than the $10 \mathrm{ng} / \sqrt{\mathrm{Hz}}$ requirement. The mechanical-thermal noise is three orders of magnitude greater than the shot noise and, while this minimum sensitivity is still good for such a small device, ignoring the mechanical-thermal noise would clearly lead to exaggerated performance claims.

Fortunately, Kenny et al. [5], measured the noise from this accelerometer, although the noise is dominated by $1 / f$ noise. At about $2 \mathrm{kHz}$, the noise makes a transition from $1 / f$ to an increase with frequency associated with a resonance in the electrostatic deflection system. The measured level-2 $\times 10^{-13} \mathrm{~A} / \sqrt{\mathrm{Hz}}-$ at this minimum is still probably due to the $1 / f$ noise but it is not out of line with the predicted mechanical-thermal equivalent current noise of $1.4 \times 10^{-13} \mathrm{~A} / \sqrt{\mathrm{Hz}}$. (Note: since $2 \mathrm{kHz}$ is well above the resonance frequency of $225 \mathrm{~Hz}$, (5) must be used to calculate the equivalent displacement noise, from which the current noise can be computed.)

As Kenny et al. demonstrate, the $1 / f$ noise can be reduced by applying force-balance feedback, although extending the feedback bandwidth beyond the resonance frequency leads to instability because of the mechanical $\pi / 2$ phase shift introduced by the mass-spring system. Alternatively, the $1 / f$ noise can be virtually eliminated by using a heterodyne detection scheme. Consequently, the device is fundamentally limited by mechanical-thermal noise. In order to drop this level to the "quiet" ocean ambient level of $10 \mathrm{ng} / \sqrt{\mathrm{Hz}}$, the displacement noise would have to be dropped to $5 \times 10^{-14} \mathrm{~m} / \sqrt{\mathrm{Hz}}$. This would require a mechanical resistance of $0.00054 \mathrm{~N}$. $\mathrm{s} / \mathrm{m}$ or a $Q$ of about 80 . Since an accelerometer can be enclosed in a completely rigid case (unlike a pressure sensor), the case can be used to withstand hydrostatic pressure and the sensor itself can be evacuated, which should produce a sufficiently high $Q$.

One proposed equivalent circuit [30] for an electrontunneling accelerometer is shown is Fig. 5. The mechanical representation includes the mass of the case $m_{c}$, in addition to the damped spring-mass system $(R, k, m)$. The accelerometer is driven by a constant-velocity source but that could be replaced by a voltage source representing constant pressure drive if the sensor were immersed in a 

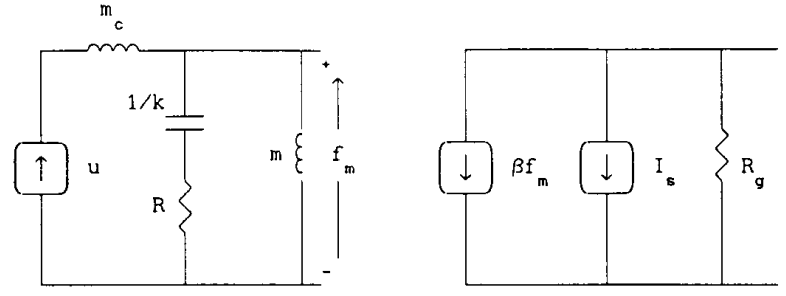

Fig. 5. Electromechanical equivalent circuit for electon-tunneling accelerometer.

fluid (as a hydrophone would be). If necessary, the radiation load can also be included as in Fig. 4, but, for noise analyses, the radiation resistance for the accelerometer is even less significant below several kilohertz than for the pressure sensor: The radiation resistance for an accelerometer goes as frequency to the fourth power [31].

The transfer constant $\beta$ (force to current) is given by

$$
\beta=I_{0} / \omega_{0}^{2} m x_{0} \quad[\mathrm{~A} / \mathrm{N}] .
$$

Notice that the mechanical resistance does not appear in the output branches. The thermal noise from this resistance enters the output circuit through $f_{m}$. Since electron tunneling is nonreciprocal [32], the amplifier current noise does not feed back into the mechanical circuit so the amplifier-induced noise is not as large as it would be for a reciprocal transducer. This advantage is inconsequential though, if the device is dominated by thermal noise in the mechanical circuit.

As the preceeding examples illustrate, mechanicalthermal noise can have observable consequences in micromachined sensors. The gross effects can be estimated easily either through Nyquist's Relation or the Equipartition Theorem. Whenever a high-sensitivity micromachined sensor is being designed, an analysis of mechanical-thermal noise should be included at an early stage to avoid being trapped with an unacceptably high noise floor.

\section{REFERENCES}

[1] 1991 Int. Conf. on Solid-State Sensors and Actuators, Dig. Tech. Pap., New York: IEEE, 1991.

[2] 1990 Solid-State Sensor and Actuator Workshop, Tech. Dig., New York: IEEE, 1990.

[3] K. E. Petersen, "Silicon as a mechanical material,"' Proc. IEEE, vol, 70 , pp. $420-457,1982$

[4] D. Hohm and G. Hess, "A subminiature condenser microphone with silicon nitride member and silicon backplate," J. Acoust. Soc. Amer., vol. 85 , pp. $476-480,1989$.

[5] T. W. Kenny, S. B. Waltman, J. K. Reynolds, and W. J. Kaiser, "Micromachined silicon tunnel sensor for motion detection,"' Appl. Phys. Lett., vol. 58, pp. 100-102, 1991.

[6] G. E. Uhlenbeck and S. Goudsmit, "A problem in Brownian motion," Phys. Rev., vol. 34, pp. 145-151, 1929.

[7] V. Tarnow, "The lower limit of detectable sound pressure," $J$. Acoust. Soc. Amer., vol. 82, pp. 379-381, 1987.

[8] C. Misner, K. Thorne, and J. Wheeler, Gravitation. New York: Freeman, 1973.

[9] G. E. Uhlenbeck and L. S. Ornstein, "On the theory of the Brownian Motion,"'Phys. Rev., vol. 36, pp. 823-841, 1930.
[10] J. W. Young, "Optimization of acoustic receiver noise performance,' J. Acoust. Soc. Amer., vol. 61, pp. 1471-1476, 1977.

[11] H. B. Callen and T. A. Welton, "Irreversibility and generalized noise," Phys. Rev., vol. 83, no. 1, pp. 34-40, 1951.

[12] F. W. Sears and G. L. Salinger, Thermodynamics, Kinetic Theory, and Statistical Thermodynamics. Reading, MA: Addison-Wesley, 1975.

[13] C. Kittel, Elementary Statistical Physics. New York: Wiley, 1958.

[14] T. J. Hofler and S. L. Garrett, "Thermal noise in a fiber optic sensor," J. Acoust. Soc. Amer., vol. 84, no. 2, pp. 471-475, 1988.

[15] A. J. Dekker, H. Hickman, and T. M. Chen, "A tutorial approach to the thermal noise in metals," Amer. J. Phys., vol. 59, pp. 609613,1991

[16] J. B. Starr, "Squeeze-film damping in solid-state accelerometers," in Solid-State Sensor and Actuator Workshop, Tech. Dig., New York, IEEE, 1990 .

[17] J. Bergqvist, F. Rudolf, J. Maisana, F. Parodi, and M. Rossi, “A silicon condensor microphone with a highly perforated backplate,' in 1991 Int. Conf. on Solid-State Sensors and Actuators, Dig. Tech. Papers, New York, IEEE, 1991, pp. 266-269.

[18] A. J. Zuckerwar, "Theoretical response of condensor microphones," J. Acoust. Soc. Amer., vol. 64, pp. 1278-1285, 1978.

[19] L. E. Kinsler, A. R. Frey, A. B. Coppens, and J. V. Sanders, Fundamentals of Acoustics, 3rd. ed. New York: Wiley, 1982.

[20] H. Chau and K. D. Wise, "Noise due to Brownian motion in ultrasensitive solid-state pressure sensors," IEEE Trans. Electron Devices, vol. ED-24, pp. 859-865, 1987.

[21] - , "Scaling limits in batch-fabricated silicon pressure sensors,' IEEE Trans. Electron Devices, vol. ED-34, pp. 850-858, 1987.

[22] H. L. Anderson, Ed., A Physicist's Desk Reference. New York: Amer. Inst. Physics, 1989.

[23] V. Tarnow, "Thermal noise in microphones and preamplifiers,' Brüel and Kjaer Tech. Rev., vol. 3, pp. 3-14, 1972.

[24] K. C. T. Ngo, "Measurement of thermal noise in condensor microphones in a vacuum-isolation vessel," M.S.E.E. thesis, Old Dominion University, Norfolk, VA, Nov. 1990.

[25] Z. van Zelst, "Circuit for condensor microphones with low noise level,"' Philips Tech. Rev., vol. 9, pp. 357-363, 1947-1948.

[26] E. L. R. Corliss and W. B. Penzes, "Low noise broadband modulated preamplifiers for a variety of transducers," Appl. Acoust., vol. 16, pp. 67-74, 1983.

[27] T. B. Gabrielson, "Fundamental noise limits in miniature acoustic and vibration sensors," Naval Air Warfare Center Rep. NADC91113-50, Dec. 31, 1991.

[28] J. J. Bernstein, "A micromachined condenser hydrophone," presented at the IEEE Solid-State Sensor and Actuator Workshop, Hilton Head, SC, June 21-25, 1992.

[29] G. Binnig and H. Rohrer, "Scanning tunneling microscopy," IBM J. Res. Develop., vol. 30, pp. 355-369, 1986.

[30] M. F. Bocko, "The scanning tunneling microscope as a high-gain, low-noise displacement sensor," Rev. Sci. Instrum., vol. 61, pp.3763-3768, 1990.

[31] P. M. Morse and K. U. Ingard, Theoretical Acoustics. Princeton, NJ: Princeton Univ. Press, 1968.

[32] M. F. Bocko, K. A. Stephenson, and R. H. Koch, "Vacuum tunneling probe: A nonreciprocal, reduced-back-action transducer," Phys. Rev. Lett., vol. 61, pp. 726-729, 1988.

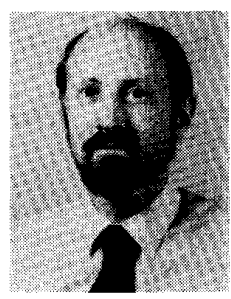

Thomas B. Gabrielson received the B.S.E.E. degree from the New Jersey Institute of Technology, Newark, and the M.Eng. and Ph.D degrees from Pennsylvania State University, University Park.

$\mathrm{He}$ has worked at the Naval Air Development Center since 1974 doing both experimental and theoretical research in underwater acoustic propagation and transduction. Presently, he is head of the Center's acoustic propagation modeling group, he directs experimental investigations in very-lowfrequency propagation, and he is involved in development of thermoacoustic projects and in evaluation of advanced-technology hydrophones 